\title{
Pulverized Oral Nicotine Equivalent to One Cigarette Reduces Parasympathomimetic Neurovegetative Tone and Increases the Physical Dependence in Smokers
}

\author{
Susana Calvo Pascual ${ }^{1 *}$, Rafa Peris Cardell ${ }^{2}$ and José Belda Ramírez ${ }^{2,3}$ \\ ${ }^{1}$ Mutua Terrassa Universitari Hospital, Plaça Dr. Robert, Terrassa, Barcelona, Spain \\ ${ }^{2}$ Arnau of Vilanova Hospital, Sant Clement Street, Valencia, Spain
}

${ }^{3}$ Medicine Department (School of Medicine), Catholic University of Valencia San Vicente Mártir, Valencia, Spain

\begin{abstract}
Introduction: Nicotine has well known long term neurovegetative effects but their relationship to tobacco dependence is unknown. We study the change in the neurovegetative tone induced after sprayed nicotine, equivalent to one cigarette in smokers and non-smokers, and its relationship of this change with smoking addiction.
\end{abstract}

Methods: 45 volunteers (33 smokers and 12 non-smokers) were included and recorded smoking history and dependence, exhaled carbon monoxide (COe), urine cotinine and electrocardiogram for the Heart Rate Variability (HRV), before and after two puffs of nicotine spray.

\begin{abstract}
Results: At baseline smokers had less HRV average difference 29 (7) $\mathrm{ms}$ and an increase of the sympathetic tone (LF: Low Frequency) 1276 (420) compared to non-smokers with high nicotine dependence 6.12 (6.20) in the Fagerström Test for Nicotine Dependence (FTND). After 5 minutes of nicotine, the HRV decreased together with a reduction in parasympathetic tone as expressed by HF (High Frequency) in smokers and non-smokers (ANOVA repeated measures $0.179(p=0.005)$ and $1.86(p=0.015)$, the LF tended to increase. These changes in parasympathetic tone were related to FTND (square $r$ lineal $0.32, p<0.05$ ) adjusted by age and sex.

Conclusions: Oral nicotine reduced immediately HRV and parasympathetic tone resulting in predominance of sympathetic tone in smokers and non-smokers, independently of smoking history and related to physical tobacco dependence. This effect could be of interest in the smoking cessation programs.
\end{abstract}

\section{Keywords}

Heart rate variability, Smoking, Nicotine, Autonomic nervous system, Oral spray

\section{Introduction}

Inhaled nicotine is the substance contained in the tobacco which produces smoking addiction. Nicotine is an organic compound of the alkaloids family, which selectively binds to the acetylcholine receptors in the autonomic ganglia, adrenal cortex and brain (cerebral cortex, hippocampus, amygdale, ventral tegmental area and nucleus accumbens) [1].

Nicotine enhances cognitive behaviours in the central nervous system related to surveillance, warning and cognitive performance, and also has a reinforcing effect on the limbic system, mediated by the neuronal pleasure pathway [2]. Intravenous administration activates adrenergic systems inducing cardiovascular effects as peripheral vasoconstriction, tachycardia, and hypertension related to its effects on the sympathetic nervous system [3].
Non-invasive study of the autonomic nervous system (ANS) can be made through the Heart Rate Variability (HRV), which is considered a quantitative marker of the balance sympathetic-parasympathetic. HRV is defined as the variation that happens in the time interval among consecutive beats [4]. HRV has been related with health status. Thus, subjects

*Corresponding author: Susana Calvo Pascual, Mutua Terrassa Universitari Hospital, Plaça Dr. Robert, 5- 08221 Terrassa, Padilla Street 237, 3-3. 08013, Barcelona, Spain

Accepted: May 03, 2021

Published online: May 05, 2021

Citation: Calvo Pascual S, Peris Cardell R, Belda Ramírez J (2021) Pulverized Oral Nicotine Equivalent to One Cigarette Reduces Parasympathomimetic Neurovegetative Tone and Increases the Physical Dependence in Smokers. Ann Pulmonol 5(1):50-55 
Citation: Calvo Pascual S, Peris Cardell R, Belda Ramírez J (2021) Pulverized Oral Nicotine Equivalent to One Cigarette Reduces Parasympathomimetic Neurovegetative Tone and Increases the Physical Dependence in Smokers. Ann Pulmonol 5(1):50-55

with reduced HRV after acute myocardial infarction had high risk of death [5]. Athletes have more HRV than sedentary people [6] and HRV decrease in a stressful situation. Some authors stated that HRV could be used to assess diabetic neuropathy, had prognostic value in patients with a severe heart attack, and it would be related to the cardiovascular risk and sudden death [7]. Also, it is well known that sympathetic stimulation would cause tachycardia and decrease HRV. On the other hand, parasympathetic stimulation releases acetylcholine causing bradycardia and increasing HRV [4].

In the 90s some studies related HRV and smoking. Thus, smoking would cause a temporary chronic decrease on the vagal control which would be related to a decrease in the HRV. This connection between smoking and HRV decrease could explain why smokers present a bigger risk of cardiovascular events $[8,9]$. This effect would be mediated by nicotine. It is thought that the continuous consumption of nicotine as stimulator of the ANS, mainly sympathetic, would be responsible for the decrease in HRV. Accordingly, it has been described that more severe tobacco consumption would be related to a bigger reduction of HRV but it was not found a relationship with the tobacco dependence [10]. However, nicotine effect could be time-limited and reinforced after each cigarette. Only few studies have evaluated the immediate effects of inhaled nicotine on HRV [11], and none about whether these effects are different between non-smokers and smokers exposed to the long-term use of nicotine.

The aim of this study was to assess the change induced by $2 \mathrm{mg}$ of oral sprayed nicotine, equivalent to one cigarette, on the heart rate variability after 5 minutes of inhalation in active smokers and in non-smokers subjects and the relationship of this change in HRV with tobacco addiction in smokers. The differences between both groups were also analyzed.

\section{Material and Methods}

\section{Subjects}

We recruited 45 consecutive volunteers from the hospital smoking cessation clinic and hospital staff. 33 smokers and 12 non-smokers subjects as the control group. The smokers were between 36-72 years old with a mean age of $51 \pm 10$ years, $54 \%$ women. The non-smokers were $51 \pm 16$ years old, $50 \%$ women. None of the participants presented serious acute pathology at the inclusion moment or other addictions besides nicotine.

The sample size was estimated in 40 subjects based on an expected mean difference between before and after nicotine inhalation of 30 (SD of 20) in the variable RMSSD which globally represent the heart rate variability as described in other studies.

\section{Study design}

The study was designed as experimental of repeated measures before-after and controlled with non-smokers individuals. All of them signed a written consent form and the study was approved by the Clinical Investigation and Ethics Committee of the Arnau de Vilanova Hospital, Valencia (Spain). Smoker patients were invited to participate in the first visit of their smoking cessation program. If they accepted, in this same visit was recorded their smoking history in cigarettes per day (CPD) and the pack year smoking index PYSI (CPD/20*years) in packs/year. Also participants took the following tests for evaluation of their addiction: Fagerström test of physical dependence to nicotine (Fagerström Test for Nicotine Dependence: FTND) [12], time to the first cigarette, the hardness score and test of psychosocial and behavioural (Glover-Nilsson Smoking Behavioural Questionnaire: GN-SBQ) [13]. A urine sample was requested from them to determine cotinine level and carbon monoxide exhaled (COe) level was measured, as well as their vital signs. An electrocardiogram record (ECG) of at least 5 minutes was digitally stored to determine the basal HRV. Next, they received two nicotine puffs $\left(2 \mathrm{mg}\right.$ ) from the oral spray (Nicorette ${ }^{\circledR}$ BucoMist,1 mg/puff) and other 5 minutes of ECG record were stored.

\section{Analysis of HRV}

The heart rate was registered with an electrocardiograph included in the electrical impedance (CardioScreen $1000^{\circledR}$, medis Medizinische Messtechnik GmbH, Germany). The signal was exported as an Excel format file (Microsoft Office ${ }^{\circledR}$, USA) for its subsequent analysis. The HRV analysis was done with the software $\operatorname{Artiifact}^{\circledR}$ [14]. This software identified semi-authomatically the interbeats intervals and edited them for manual inspection according to the recommendations published in the international guides [15]. Later, HRV variables were calculated. The heart rate registers were only considered valid from at least 300 consecutive seconds and if they had no more than $20 \%$ of interpolated beats from the total recorded beats. The spectral components of the frequency domain registered were:

- High frequency (HF $\left.\left(\mathrm{ms}^{2}\right)\right)$ defined as the power in the frequencies range from $0.15 \mathrm{~Hz}$ to $0.4 \mathrm{~Hz}$.

- Low frequency (LF $\left(m s^{2}\right)$ ) defined as the power in the frequencies range from $0.04 \mathrm{~Hz}$ to $0.15 \mathrm{~Hz}$.

- $\quad$ and the relationship between both: LF /HF.

And the domain components were:

- The standard deviation of the intervals R-R ((standard deviation of normal- to-normal intervals: SDNN (ms))

- The square root of the average of the sum differences square between the intervals R-R adjacent (root mean square of the successive differences RMSSD (ms)).

\section{Statistical analysis}

The results were tabulated and stored in an Excel file (Microsoft Office ${ }^{\circledR}$, USA). The continuous variables were expressed as mean \pm standard deviation (SD) or median (interquartile range). Categorical variables were expressed as number (percentage). Variables of exponential growth were expressed as logarithms in base 10 . Comparisons between groups of the basal characteristics were done with Student's t-test for no matched data or the Mann-Whitney $U$ test. Repeated measures analysis of variance was used to assess the changes in the variables before and after nicotine sprayed. The study of the relationship between the changes in the var- 
Citation: Calvo Pascual S, Peris Cardell R, Belda Ramírez J (2021) Pulverized Oral Nicotine Equivalent to One Cigarette Reduces Parasympathomimetic Neurovegetative Tone and Increases the Physical Dependence in Smokers. Ann Pulmonol 5(1):50-55

Table 1: Description of the vital signs in smokers and non-smokers, before and after oral spray of nicotine.

\begin{tabular}{|l|l|l|l|l|}
\hline & \multicolumn{2}{|l|}{ Smokers $(\mathbf{n}=33)$} & \multicolumn{2}{l|}{ Non smoker $(\mathbf{n}=12)$} \\
\hline & Mean & Stand. Dev. & Mean & Stand. Dev. \\
\hline SBP1 & 130.67 & 20.90 & 125.92 & 11.48 \\
\hline SBP2 & 136.89 & 17.54 & 137.50 & 17.89 \\
\hline DBP1 & 78.67 & 10.74 & 69.00 & 7.71 \\
\hline DBP2 & 81.21 & 9.39 & 80.50 & 9.37 \\
\hline HR1 & 73.36 & 10.17 & 64.63 & 7.02 \\
\hline HR2 & 77.03 & 9.80 & 76.71 & 9.06 \\
\hline OS1 & 95.67 & 1.45 & 96.50 & 1.38 \\
\hline OS2 & 96.15 & 1.61 & 96.58 & 1.62 \\
\hline
\end{tabular}

SBP: Systolic blood pressure; DBP: Diastolic blood pressure; HR: Heart rate; OS: oxygen saturation.

Numbers 1 and 2 in the same variables are before and after oral spray of nicotine.

Table 2: Mean and standard deviation of Heart Rate Variability on the time domain and the frequency domain of smokers and non smokers before and after nicotine pulverized oral.

\begin{tabular}{|l|l|l|l|l|}
\hline & \multicolumn{2}{|l|}{ Smokers (n $\mathbf{3 3})$} & \multicolumn{2}{l|}{ Non smokers (n 12) } \\
\hline & Mean & Stand. Dev. & Mean & Stand. Dev. \\
\hline RMSSD1 & 64.07 & 25.31 & 34.70 & 18.35 \\
\hline RMSSD2 & 63.50 & 27.37 & 24.33 & 16.81 \\
\hline LF (abs)1 & 2000.87 & 2249.34 & 724.46 & 404.20 \\
\hline LF (abs)2 & 2295.20 & 2571.78 & 786.39 & 806.58 \\
\hline HF (abs)1 & 717.62 & 566.33 & 554.87 & 699.85 \\
\hline HF (abs)2 & 712.90 & 538.59 & 342.48 & 575.25 \\
\hline LF/HF1 & 2.71 & 1.59 & 3.79 & 4.68 \\
\hline LF/HF2 & 3.30 & 2.83 & 4.49 & 2.60 \\
\hline
\end{tabular}

RMSSD: The square root of the average of the sum differences square between the intervals R-R adjacent; LF (abs): Low Frequency component of the HRV with absolute numbers; HF (abs): High Frequency component of the HRV with absolute numbers.

The numbers 1 and 2 shown pre and post nicotine pulverized oral.

iables HRV and variables of tobacco dependence were analyzed by multivariate linear regressions. Statistical significance was established in 0.05 and all the analysis were done with the statistical software SPSS (version 15.0, SPSS Inc., Chicago, USA). Sample size calculations were performed with GPower 3.1.9.7 (Düsseldorf, Germany).

\section{Results}

All participants successfully completed the study and 45 subjects were included, 33 smokers and 12 non-smokers as a control group.

In the smokers group $88 \%$ of them had stable pathologies (cardiovascular $36 \%$, respiratory $27 \%$, others $37 \%$ ) and until $76 \%$ of them suffered from respiratory symptoms. In the non-smokers group $50 \%$ of them had stable pathologies (cardiovascular $42 \%$, respiratory $17 \%$, others $41 \%$ ) and $8 \%$ had respiratory symptoms. Vital signs are shown on Table 1. Blood pressure and heart rate increased significantly after spraying nicotine in both groups.

Tobacco consumption data and the physical and psychical dependence of the smokers were cigarettes per day (mean (SD)) $22.45 \pm 8.45$, the packs year index $37.27 \pm 16.04$ packs/ year, Heavy Smoking Index (HSI): $3.70 \pm 1.26$, Fagerström Test for Nicotine Dependence (FTND): $6.12 \pm 6.20$, Glover-Nilsson Smoking Behavioural Questionnaire (GN-SBQ): $17.81 \pm 8.60$, cotinine in urine: $7558 \pm 4118 \mathrm{ng} / \mathrm{ml}$ and expired air carbon monoxide measurement: $21 \pm 9$. The time to smoke the first cigarette after getting up in the morning was less than 5 minutes $51.5 \%$ of them, between 5 and 30 minutes $36.4 \%$ and more than 30 minutes $12.1 \%$.

The results of the analysis of HRV variables are shown in Table 2. When smokers and non smokers were compared, a significant difference was found in the variables RMSSD and LF (average difference (standard error: SE ) 29 (7) ms; $p<0.0001$ and 1276 (420); $\mathrm{p}=0.005 \mathrm{~ms}^{2}$ respectively). This suggests that smokers have less HRV and an increase of the sympathetic tone regarding to non smokers as described elsewhere.

After spraying nicotine into the mouth the HRV variables of the time domain decreased with a significant difference in RMSSD (transformed into base 10 logarithm) repeated measurements analysis of variance $0.179(p=0.005)$ and an inter-subjects effect for tobacco use of $0.152(p=0.010)$. This indicates that nicotine produced a lighter decrease of more intensity in non smokers than in smokers. For the variables of frequency domain an increase was observed in the average $\mathrm{LF}$ and LF/HF and a decrease in HF, being significant only for $\mathrm{HF}$ (transformed into base 10 logarithm) with a LF = 1,86, $p=$ 0.015 , the drop was more marked for non smokers than for smokers (see Figure 1). In the variables LF and LF/HF there was a tendency but it did not become too significant. All results were adjusted by age, cardiovascular history, and sex which influence directly with the ANS with no changes.

These results suggest than nicotine decreases in less than $5 \mathrm{~min}$ the HRV and induces a reduction in the parasympathetic tone remaining the sympathetic tone as dominant. This effect was appreciated in smokers as well as non smokers, which suggests that the acute effect of the oral nicotine sprayed in the HRV and the autonomic vegetative system does not disappear in people used to nicotine use but, maybe, it is of less intensity.

The relationship between the variables of tobacco use and dependence with the HRV variables was assessed by multivariate regression analysis. There was a significant relationship between the change in the HF (as logarithm) and the physical dependence when adjusted by age and sex $(R=0.68$, $\mathrm{R}^{2}=0.46$ with a $\mathrm{p}=0.009$ ).

The following equation was obtained:

FTND $=8.3-0.2($ age $)+1.6($ sex $)+0.1($ PYSI $)+1.8$ (change in $\log 10(\mathrm{HF}))$

Some side effects were observed from oral spray (Nicorette ${ }^{\circledR}$ Bucomist). The most frequent effect was hiccup and other minor effect as described in the data sheet of the 


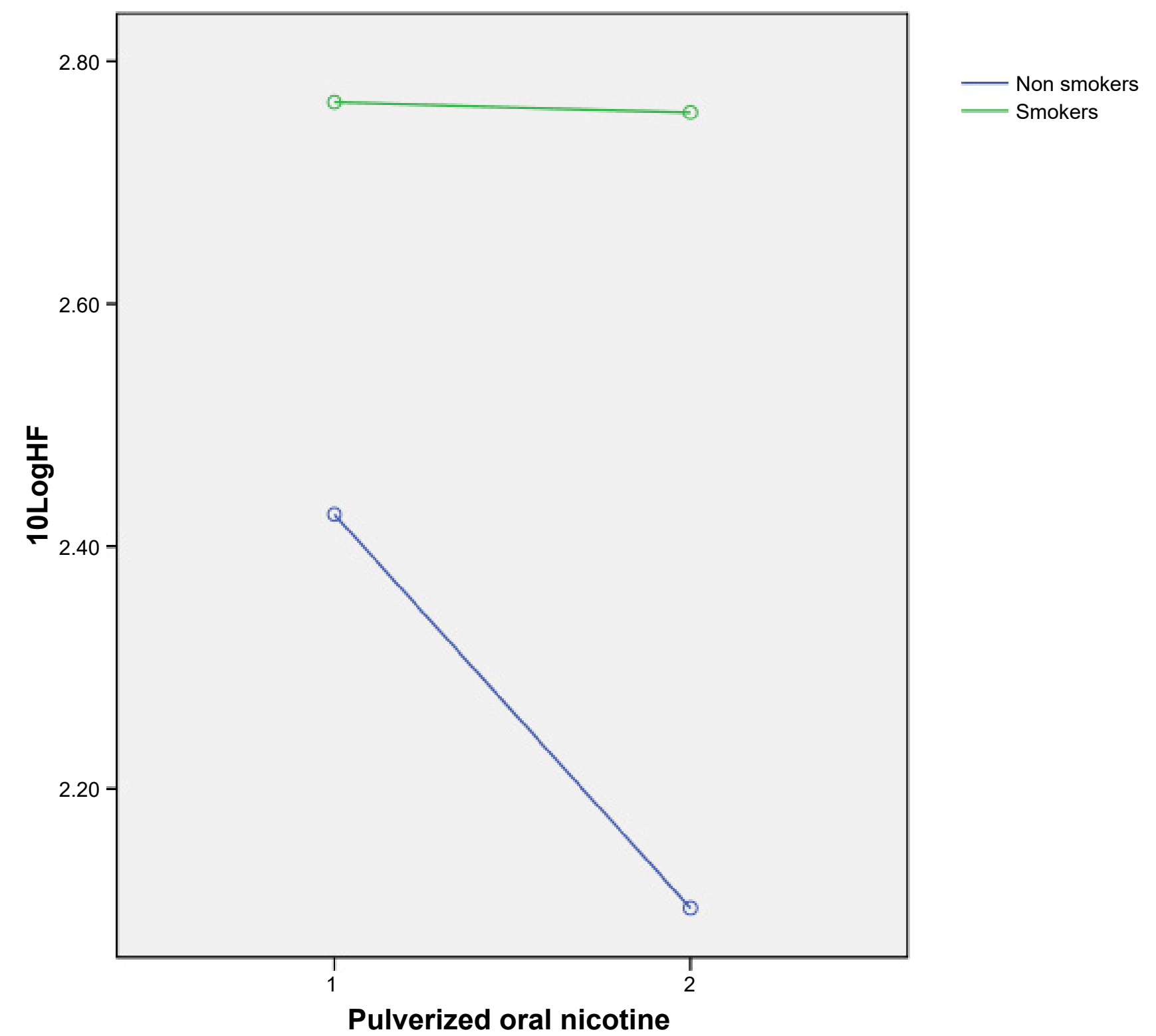

10LogHF: base 10 logarithm of the frequency domin HF: High Frequency. 1: before2mg of nicotine, 2: after $2 \mathrm{mg}$ of nicotine

Figure 1: Pulverized oral of nicotine, $2 \mathrm{mg}$ in smoker sand non smokers.

product. One patient had inconveniences, not clear pain, in the left upper limbs with sweating and nauseas which was oriented as angina pectoris, but it was self- limited without treatment (woman of 74 years old, non smoker with long history of ischemic cardiopathology). Curiously, some subjects complaint of tearing and cough [16].

\section{Discussion}

Many studies have shown that nicotine has an important effect on the ANS but this is the first study showing that this effect is related to physical dependence, suggesting that in one particular subject, the more the parasympathetic tone is reduced by nicotine, the greater physical dependence presents this subject.
The results shown as a novelty that immediately after each cigarette in less than 5 minutes, nicotine produced a combined effect on the ANS promoting sympathetic tone dominance. This effect was not only by directly stimulating the sympathetic but mainly decreasing parasympathetic tone. The magnitudes of these acute changes on the parasympathetic tone after nicotine were related to physical dependence as assessed by FTND.

At our best knowledge there are not any published studies showing significant relationship between nicotine effects on HRV and nicotine dependence. Soares Dos Santos, et al. [10] analyzed the HRV relationship with FNTD in smokers but it was not significant. Similarly we did not found relationship 
Citation: Calvo Pascual S, Peris Cardell R, Belda Ramírez J (2021) Pulverized Oral Nicotine Equivalent to One Cigarette Reduces Parasympathomimetic Neurovegetative Tone and Increases the Physical Dependence in Smokers. Ann Pulmonol 5(1):50-55

between HRV and FNTD in smokers at baseline. However, when we considered the change after nicotine sprayed this relationship became obvious. We could suppose that FNTD is relatively independent of the HRV that any smokers had at any time but it is strongly dependent of the magnitude of the change that nicotine had after every single cigarette. In other words, physical dependence to nicotine seems a function of the neurovegetatives changes that nicotine induces with every smoked cigarette.

The tobacco psychical dependence in smokers, and therefore psychosocial and behavioural dependence, would be more related to the dopaminergic system. This could explain why HRV and so ANS modulation is modified mostly with physical dependence and non with psychical dependence. Neurobiological mechanisms responsible for positive reinforcement generated by nicotine have their seat in dopaminergic neurons of mesolimbic system [17]. Available pharmacological and psychological treatments as well as Cognitive Behavioural Therapy could modify this pathway [18]. Smoking behaviour is even more complex as the Brazilian study described, thus smokers with higher nicotine dependence presented less extroverted personality [19]. In rats, it was shown that anxiety behaviour and nicotine dependence were strongly related [20]. However, physical and psychical dependence are connected as suggested by the low vagal reactivity observed in smokers in response to stress [21], which implies a reduced capacity of the individual to resist smoking desire. This study and our results suggest that maybe treating parasympathetic tone will be a good smoking cessation strategy.

The HRV is an old technique well described but not clear reference values are available due to the many analysis and variables that can be done and used. The HRV values for smokers and non smokers registered in this study were very similar to others described in several reference populations $[22,23]$ using ECG records of 20 and 5 minutes, respectively, with the same target variables.

The baseline results obtained were congruent with many other studies $[24,25]$ in showing that smokers had less HRV. Several authors also described that after smoking one cigarette decreased HRV from 3 to 30 minutes later $[8,26,27]$. Another study [28] using double dose of nicotine $(4 \mathrm{mg})$ in oral pills showed that nicotine decreased the HRV and significantly modified the components of frequency domain favouring the sympathetic dominance in healthy non-smokers. Our results presented here showed that this effect also happened in non-smokers as well as smokers but of less intensity, which is congruent with the effect of nicotine on HRV described in the passive smokers [29].

The nicotine dose sprayed in the present study, $2 \mathrm{mg}$, was similar to smoke one cigarette according to Hansson [30]. Therefore, it was assumed that the effects found could be equivalent to a cigarette smoked. Although it was not fully proved in this study, it could be speculated that nicotine effects in smokers were similar to non-smokers but perhaps of less intensity. And these effects were not lost after many years of exposure to nicotine and possibly neither after successive cigarettes they smoked daily. We checked that by analyzing the relationship between the changes after nicotine and the PYSI and the cotinine and COe levels. Not significant relationships were obtained suggesting that there was not lost of effect depending of the pack years smoked nor since the last cigarette smoked.

This study has some limitations. One limitation is related to the kind of subjects recruited, and the pathologies they had, which could influence in HRV results. However, we considered that repeated measures analyses on each patient could deal with that. Another limitation would be the sample size. The calculations were performed for the main HRV variable in the time domain like other similar studies. This assumption could not be sufficient for all HRV variables and could explain that LF did not get significant. That did not invalidate the results shown in the paper but nicotine also may have some minor effects on other HRV variables.

In conclusion, oral nicotine pulverized in smokers and non-smokers produced a significant and immediate reduction in the HRV and an effect on the neurovegetative balance, leaving to a sympathetic predominance by reducing the HF component (parasympathetic tone) which was related to physical dependence to nicotine. Treatment addressed to modify this parasympathetic effect of nicotine could be of interest in the smoking cessation strategies.

\section{Conflict of Interests}

The authors declare not having any conflict of interests.

\section{References}

1. Dani JA, Bertrand D (2007) Nicotinic acetylcholine receptors and nicotinic cholinergic mechanisms of the central nervous system. Annu Rev Pharmacol Toxicol 47: 699-729.

2. Subramaniyan M, Dani JA (2015) Dopaminergic and cholinergic learning mechanisms in nicotine addiction. Ann N Y Acad Sci 1349: 46-63.

3. Takashi N, Kosuke F, Hiroyuki K, et al. (2019) The effects of transdermal nicotine patches on the cardiorespiratory and lactate responses during exercise from light to moderate intensity: Implications for exercise prescription during smoking cessation. Medicina (B Aires) 55: 348.

4. Gallo JA, Farbiarz J, Álvarez DL (1999) Análisis espectral de la frecuencia cardíaca. latreia 12: 61-71.

5. Wolf MM, Varigos GA, Hunt D, et al. (1978) Sinus arrhythmia in acute myocardial infarction. Med J Aust 2: 52-53.

6. Arai Y, Saul J, Albrecht P, et al. (1989) Modulation of cardiac autonomic activity during and immediately after exercise. Am J Physiol 256: 132-141.

7. Karim N, Hasan JA, Ali SS (2011) Heart rate variability-A review. J Basic Appl Sci 7: 71-77.

8. Hayano J, Yamada M, Sakakibara Y, et al. (1990) Short-and longterm effects of cigarette smoking on heart rate variability. Am J Cardiol 65: 84-88.

9. Unverdorben M, Von HK, Winkelmann BR (2009) Smoking and atherosclerotic cardiovascular disease: Part III: Functional biomarkers influenced by smoking. Biomark Med 3: 807-823.

10. Soares SAP, Ramos D, Martins OG, et al. (2016) Influence of 
Citation: Calvo Pascual S, Peris Cardell R, Belda Ramírez J (2021) Pulverized Oral Nicotine Equivalent to One Cigarette Reduces Parasympathomimetic Neurovegetative Tone and Increases the Physical Dependence in Smokers. Ann Pulmonol 5(1):50-55

smoking consumption and nicotine dependence degree in cardiac autonomic modulation. Arq Bras Cardiol 510-518.

11. Dinas PC, Koutedakis Y, Flouris AD (2013) Effects of active and passive tobacco cigarette smoking on heart rate variability. Int J Cardiol 163: 109-115.

12. Jiménez RCA, KO FK (2003) ¿'Hacemos bien el test de Fagerström? Prev Tab 5: 161-162.

13. Glover ED, Nilsson F, Westin A, et al. (2005) Developmental history of the glover-nilsson smoking behavioural questionnaire. Am J Heal Behav 29: 443-455.

14. Kaufmann T, Sütterlin S, Schulz SM, et al. (2011) ARTiiFACT: A tool for heart rate artifact processing and heart rate variability analysis. Behavior Research Methods 43: 1161-1170.

15. (1996) Heart rate variability. Eur Heart J 17: 354-381.

16. AEMPS (2012) Nicorette BucoMist. Ficha técnica. Agencia Española Medicam y Prod Sanit.

17. Wise RA (2004) Dopamine, learning and motivation. Nat Rev Neurosci 5: 483-494.

18. De Higes EBPL (2015) Manual separ de procedimientos: Manejo diagnóstico y tratamiento del tabaquismo en la práctica clínica diaria.

19. De Cássia R, Botelho C, Da Silva AMCGR (2003) Características de personalidade e dependência nicotínica em universitários fumantes da UFMT. J Pneumol 29: 21-27.

20. Casarrubea M, Pierucci M, Aiello S, et al. (2020) Effects of chronic nicotine on the temporal structure of anxiety-related behaviour in rats tested in hole-board. Prog Neuro-Psychopharmacology Biol Psychiatry 96: 109731.
21. Ashare RL, Sinha R, Lampert R, et al. (2012) Blunted vagal reactivity predicts stress-precipitated tobacco smoking. Psychopharmacology 220: 259-268.

22. Murgia F, Melotti R, Foco L, et al. (2019) Effects of smoking status, history and intensity on heart rate variability in the general population: The CHRIS study. PLoS One 14: e0215053.

23. Dantas ME, Dias daSVJ, Martins I, et al. (2018) Reference values for short-term resting-state heart rate variability in healthy adults: Results from the brazilian longitudinal study of adult health-ELSA-Brasil study. Psychophysiology 55: e13052.

24. Pereira E, Ribeiro IJS, Vidal I, et al. (2017) The smoking habit negatively influences autonomic heart control in community-dwelling elderly adults. Hell Soc Cardiol 58: 283-288.

25. Sumartiningsih S, Lin HF, Lin JC (2019) Cigarette smoking blunts exercise-induced heart rate response among young adult male smokers. Int J Environ Res Public Health 16: 1032.

26. Karakaya O, Barutcu I, Kaya D, et al. (2007) Acute effect of cigarette smoking on heart rate variability. Angiology 58: 620-624.

27. Kobayashi F, Watanabe T, Akamatsu Y, et al. (2005) Acute effects of cigarrete smoking on the heart rate variability of taxi drivers during work. Scand J Work Environ Health 31: 360-366.

28. Sjoberg N, Saint DA (2011) A single $4 \mathrm{mg}$ dose of nicotine decreases heart rate variability in healthy nonsmokers: Implications for smoking cessation programs. Nicotine Tob Res 13: 369-372.

29. Arden C, Eatough D, Gold D, et al. (2001) Acute exposure to environmental tobacco smoke and heart rate variability. Environ Health Perspect 109: 711-716.

30. Hansson, et al. (2011) Alivio de la ansiedad con spray bucal de terapia de reemplazo de nicotina. In: Poster POS3-45. Presentado En SRNT. Toronto Canadá.

DOI: $10.36959 / 918 / 452$

Copyright: (C) 2021 Calvo Pascual S, et al. This is an open-access article distributed under the terms of the Creative Commons Attribution License, which permits unrestricted use, distribution, and reproduction in any medium, provided the original author and source are credited. 